\title{
Muffins adicionados de farinha de feijão de diferentes classes
}

\author{
Different classes of bean flour added to muffins
}

\section{Lúcia Fabiane Trindade de Barros ${ }^{1}$, Thomas Duzac Escobar'1, Paula Ferreira de Araújo Ribeiro', Tiago André Kaminski ${ }^{1 *}$}

${ }^{1}$ Universidade Federal do Pampa (UNIPAMPA), campus Itaqui, Itaqui/RS - Brasil

\author{
*Corresponding author \\ Tiago André Kaminski, Universidade Federal do Pampa (UNIPAMPA), Campus Itaqui, Departamento de Ciência e Tecnologia de Alimentos, Rua Luiz \\ Joaquim de Sá Britto, Promorar, CEP: 97650-000, Itaqui/RS - Brasil, e-mail: tiagokaminski@unipampa.edu.br
}

Cite as: Different classes of bean flour added to muffins. Braz. J. Food Technol., v. 21, e2017081, 2018.

Received: May 28, 2017; Accepted: Apr. 30, 2018

\section{Resumo}

O muffin consiste em uma porção individual de bolo, de grande aceitação, mas normalmente elaborado com ingredientes de elevado valor calórico, como todos os bolos; em razão disso, é empregado como veículo para incorporação de ingredientes e nutrientes que promovam o enriquecimento nutricional. Nesse sentido, o trabalho se propôs a elaborar muffins com substituição parcial da farinha de trigo por farinhas de diferentes classes de feijão (preto, vermelho, carioca e branco) e avaliar as suas propriedades físicas, a composição química e os atributos sensoriais. O muffin padrão (MP) foi feito com farinha de trigo, leite, ovos, óleo de soja, fermento químico e açúcar, como ingredientes. Nos demais, 30\% da farinha de trigo foi substituída por farinha de feijão branco (MFB), vermelho (MFV), carioca (MFC) e preto (MFP). A substituição da farinha de trigo proporcionou rendimentos superiores e menores perdas de peso no assamento, o que sugere maior capacidade de retenção de água conferida pelas farinhas de feijão, mesmo sem diferir no volume dos muffins. A substituição da farinha de trigo também acarretou maiores custos das massas cruas, proporcionalmente ao valor comercial das classes de feijão. No entanto, após o processo de assamento e considerados rendimentos e pesos finais, os custos das unidades de muffins foram semelhantes. Nutricionalmente, admite-se o incremento do valor nutricional devido ao aumento dos teores de matéria mineral, proteína, fibra alimentar e compostos fenólicos totais, em detrimento dos carboidratos digeríveis e do valor calórico. Sensorialmente, apenas MFB e MFP tiveram aceitação, intenção de compra e preferência equivalentes ao MP, mas todos os muffins apresentaram altos índices de aceitabilidade. A substituição parcial de ingredientes energéticos, como a farinha de trigo, pela de feijão, possibilita obter muffins com propriedades físicas adequadas, maior qualidade nutricional e altos índices de aceitabilidade, sem encarecer o produto final.

Palavras-chave: Phaseolus vulgaris; Rendimento; Proteína; Fibra alimentar; Compostos fenólicos; Análise sensorial.

\section{Abstract}

The muffin consists of an individual portion of cake, of great acceptance, but usually elaborated with ingredients of high caloric value, like all cakes. For this reason, the muffin has been used as a vehicle for the incorporation of ingredients and nutrients that promote nutritional enrichment. The purpose of this work was to prepare muffins with partial substitution of the wheat flour by different classes of bean flour (white, red, "carioca" and black beans) and evaluate their physical properties, chemical composition and sensory attributes. The ingredients of the standard muffin (MP) were wheat flour, milk, eggs, soy oil, baking powder and sugar. For the other muffins, 30\% of the wheat flour was substituted by white bean (MFB), red bean (MFV), "carioca" bean (MFC) and black bean (MFP) flours. The substitution of wheat flour by bean flour resulted in higher yields and smaller weight losses on baking, suggesting that the bean flours conferred a greater water holding capacity without affecting the volume of the muffins. Substitution of the wheat flour also entailed higher costs of the raw doughs, in proportion to the commercial value of the bean classes. However, after the baking process and considering the yields and final weights, the costs of the muffin units were similar. Nutritionally, an increment in the nutritional values occurred with increases in the mineral matter, protein, dietary fibre and total phenolic compounds, in detriment of the digestible carbohydrates and caloric value. Regarding the sensorial attributes, only MFB and MFP showed acceptance, purchase intention and preference equivalent 
to MP, but all the muffins showed high acceptance indexes. The partial substitution of energetic ingredients, such as wheat flour, by bean flour, made it possible to obtain muffins with adequate physical properties, higher nutritional quality and high acceptance indexes, without increasing the price of the final product.

Keywords: Phaseolus vulgaris; Yield; Protein; Dietary fibre; Phenolic compounds; Sensory analysis.

\section{Introdução}

A produção mundial de feijão (Phaseolus vulgaris L.) é de 26,5 milhões de toneladas; o Brasil, com uma produção de 3,3 milhões de toneladas, compreende 12,4\% do total, menor apenas que Mianmar e Índia, com produções de 4,6 e 4,1 milhões de toneladas, respectivamente (FAO, 2014). O feijão tem pouca expressão do comércio internacional em razão da elevada demanda de consumo nos países produtores e ampla variedade de tipos de feijão, aliada às diferenças de hábitos alimentares entre países e até entre regiões; por exemplo, o feijão carioca é o mais produzido e consumido no Brasil, mas não tem boa aceitação no mercado externo (CONAB, 2015).

O feijão é uma leguminosa de alto valor proteico e baixa quantidade de lipídeos (FROTA et al., 2010). A proteína do feijão é rica no aminoácido essencial lisina; entretanto, é pobre nos aminoácidos essenciais sulfurados metionina e cisteína (VIEIRA et al., 2011). Ressaltam-se, também, teores consideráveis de fibra alimentar, carboidratos complexos, vitaminas (principalmente do complexo B) e polifenóis com propriedades antioxidantes (SILVA et al., 2009).

Dentre os produtos de panificação, o bolo se destaca como um alimento apetitoso e com alto índice de aceitação por consumidores de várias idades e classes sociais, principalmente por ser um alimento leve, de fácil mastigação e por ter uma consistência permeável, que remete à facilidade de digestão (MOSCATTO et al., 2004). Embora a composição e, consequentemente, o valor nutricional do bolo seja bastante variável, de modo geral, é um alimento com elevado teor de carboidratos, lipídeos e proteínas, fazendo com que seja um produto com elevado valor calórico. Os processos de retirada ou redução dos ingredientes mais energéticos não são simples, pois a diversidade dos ingredientes é responsável por proporcionar atributos de textura, maciez e sabor, além de conferir maior período de conservação ao alimento. No entanto, se a substituição destes ingredientes se der por outros com funções equivalentes, a obtenção de produtos menos calóricos é possível (BENASSI et al., 2001).

O muffin é um produto de origem anglo-americana, consiste em uma porção individual de bolo, de apresentação diferenciada e com enorme aceitação no mundo todo (CURREA; SIERRA, 2010; MARTÍNEZ-CERVERA et al., 2012). Assim como outros bolos, o muffin é elaborado com ingredientes de elevado valor calórico; entretanto, independentemente disso, mantém elevados índices de aceitação pelos consumidores devido ao sabor agradável e à textura macia (MARTÍNEZ-CERVERA et al., 2012).

Diversas propostas de enriquecimento nutricional têm muffins como veículos, normalmente com substituição de ingredientes por outros produtos da indústria de alimentos, tais como farinha de arroz (SEGURA et al., 2014), farelo de arroz (BIBALAN et al., 2013), farelo de mandioca desidratado (BARCELO et al., 2014), casca de uva (BENDER, 2015; BENNEMANN et al., 2016) e sorgo (POQUETTE et al., 2014). Por outro lado, os poucos trabalhos que testaram a adição de farinha de feijão em produtos de panificação, como biscoito, rocambole, bolo e macarrão, descrevem sobre formulações com apenas uma classe da leguminosa (BARCHET et al., 2010; CASAGRANDI et al., 1999; FROES, 2012; FROTA et al., 2010).

Nesse contexto, o trabalho se propôs a elaborar muffins com substituição parcial da farinha de trigo por farinhas de diferentes classes de feijão (preto, vermelho, carioca e branco), além de avaliar suas propriedades físicas, composição química e atributos sensoriais.

\section{Material e métodos}

\subsection{Ingredientes}

Farinha de trigo tipo 1 (Maria Inês, Antoniazzi e Cia Ltda.), leite integral (Piá ${ }^{\circledR}$, Cooperativa Piá), ovos (Granja Avícola Petry Ltda.), óleo de soja (Soya, Bunge), fermento em pó químico (Royal ${ }^{\circledR}$, Mondeléz International), açúcar refinado especial (Caravelas ${ }^{\circledR}$, Grupo Colombo) e feijões, de diferentes classes e mesma marca comercial (CBS ${ }^{\circledR}$, CBS Alimentos), adquiridos no comércio local da cidade de Itaqui-RS. Os grãos de feijão foram moídos em micromoinho (A11, IKA) e as frações moídas peneiradas através de peneira de 50 mesh.

\subsection{Elaboração dos muffins}

Foram elaboradas cinco formulações, sendo uma padrão com farinha de trigo e outras quatro com substituição de $30 \%$ da farinha de trigo pelas farinhas das diferentes classes de feijões, mantendo a mesma proporção para os demais ingredientes (Tabela 1).

Os produtos foram elaborados com base no trabalho de Martínez-Cervera et al. (2012), com algumas modificações. As claras dos ovos foram batidas em batedeira planetária orbital (BAT600, Cadence) por cinco minutos, acrescidas do açúcar e batidas por mais um minuto na velocidade máxima; posteriormente, adicionaram-se as gemas e metade do leite, batendo-se por um minuto na 
Muffins adicionados de farinha de feijão de diferentes classes

Barros, L. F. T et al.

Tabela 1. Custos dos ingredientes e seus percentuais nas formulações dos muffins

\begin{tabular}{|c|c|c|c|c|c|c|}
\hline \multirow{2}{*}{ Ingredientes } & Custo & MP & MFB & MFV & MFC & MFP \\
\hline & (R\$/kg) & \multicolumn{5}{|c|}{$\%$} \\
\hline Farinha de trigo & 2,34 & 100 & 70 & 70 & 70 & 70 \\
\hline Farinha de feijão branco & 11,96 & 0 & 30 & 0 & 0 & 0 \\
\hline Farinha de feijão vermelho & 10,98 & 0 & 0 & 30 & 0 & 0 \\
\hline Farinha de feijão carioca & 5,96 & 0 & 0 & 0 & 30 & 0 \\
\hline Farinha de feijão preto & 5,08 & 0 & 0 & 0 & 0 & 30 \\
\hline \multicolumn{7}{|c|}{ Proporção para a quantidade de farinha (\%) } \\
\hline Leite $^{*}$ & 2,48 & 50 & 50 & 50 & 50 & 50 \\
\hline Ovo & 7,15 & 100 & 100 & 100 & 100 & 100 \\
\hline Óleo de soja** & 2,89 & 35 & 35 & 35 & 35 & 35 \\
\hline Fermento químico & 24,90 & 2 & 2 & 2 & 2 & 2 \\
\hline Açúcar & 1,79 & 80 & 80 & 80 & 80 & 80 \\
\hline Custo de $367 \mathrm{~g}$ de massa $(\mathrm{R} \$)$ & & 1,37 & 1,61 & 1,63 & 1,48 & 1,45 \\
\hline Custo da unidade de muffin $(\mathrm{R} \$)^{\star \star *}$ & & 0,08 & 0,08 & 0,09 & 0,08 & 0,08 \\
\hline
\end{tabular}

${ }^{*} \mathrm{R} \$ 2,55$ por litro, transformado em gramas pela densidade $1,031 \mathrm{~g} / \mathrm{mL} ;{ }^{* *} \mathrm{R} \$ 2,39$ por litro, transformado em gramas pela densidade $0,92 \mathrm{~g} / \mathrm{mL}$; ${ }^{* * \star}$ Considerando as médias de rendimento e peso final de cada formulação (Tabela 2). Legenda: MP (muffin padrão), MFB (muffin feijão branco), MFV (muffin feijão vermelho), MFC (muffin feijão carioca) e MFP (muffin feijão preto).

velocidade média; seguiu-se a adição do restante do leite e do óleo de soja, aos poucos, batendo-se por mais dois minutos na velocidade média; por último, as farinhas e o fermento foram adicionados aos poucos e misturados manualmente. A massa obtida foi enformada em formas metálicas revestidas com forminhas de papel e levada ao assamento em forno elétrico (Du Chef $45 \mathrm{~L}$, ITC Eletro) pré-aquecido a $120^{\circ} \mathrm{C}$, durante 10 minutos, complementado por mais cinco minutos com o dourador ligado.

\subsection{Análises físicas}

Os pesos de 20 unidades de cada formulação foram mensurados em balança semianalítica antes e após o assamento. O rendimento em peso foi determinado pela razão entre os pesos das massas assadas e os pesos das massas cruas. A perda de peso no assamento foi avaliada pela seguinte fórmula: [(peso da massa crua - peso da massa cozida)×100]/peso da massa crua. O volume aparente dos muffins foi avaliado pelo método de deslocamento de sementes de painço (AACCI, 2001) e, a partir dos dados de volume e peso da massa cozida, foi determinado o volume específico em $\mathrm{mL} \mathrm{g}^{-1}$ de cada formulação.

\subsection{Análises químicas}

As análises foram precedidas pela pré-secagem dos muffins em estufa com circulação de ar forçado (SL 102/480, Solab) a 55 C durante 24 horas e moagem em micromoinho (A11, IKA). Foram determinados os teores de umidade, cinzas, proteína bruta e fibra alimentar (insolúvel e solúvel), conforme os métodos analíticos propostos pela Association of Official Analytical Chemists (AOAC, 2000). O teor de lipídeos foi quantificado a partir do método descrito por Bligh e Dyer (1959); a quantidade de carboidratos foi estimada pela diferença de 100 menos os parâmetros citados anteriormente, e o valor calórico foi calculado de acordo com a RDC n. ${ }^{\circ} 360$ do Ministério da Saúde (BRASIL, 2003), considerando os fatores de conversão de $4 \mathrm{kcal} \mathrm{g}^{-1}$ para carboidratos e proteínas, e de $9 \mathrm{kcal} \mathrm{g}^{-1}$ para lipídeos.

A determinação dos compostos fenólicos totais foi precedida pela extração dos mesmos, conforme metodologia descrita por Velioglu et al. (1998), com modificações. Os extratos foram obtidos a partir de $10 \mathrm{~g}$ de amostra seca macerada com $40 \mathrm{~mL}$ de solução extratora de metanol aquoso 70\% (v/v), mediante agitação por uma hora em mesa agitadora e temperatura ambiente, filtragem a vácuo em papel Whatman n. ${ }^{\circ} 1$ e ajuste do volume para $50 \mathrm{~mL}$ com a solução extratora. As concentrações foram determinadas por análise espectrofotométrica a $760 \mathrm{~nm}$, conforme metodologia de Singleton e Rossi (1965), utilizando curva padrão de ácido gálico, com sete pontos, nas concentrações de 0 a 150 ppm. Os resultados foram expressos em equivalente de ácido gálico (AGE) por $100 \mathrm{~g}$ de muffin.

Todas as análises químicas foram realizadas em três repetições para cada muffin.

\subsection{Análises sensoriais}

O estudo foi previamente submetido e aprovado pelo Comitê de Ética em Pesquisa com Seres Humanos da Universidade Federal do Pampa, mediante parecer n. ${ }^{\circ}$ 1.131.461. Foram realizados testes de aceitação, intenção de compra e preferência com provadores de ambos os sexos, não treinados, pertencentes à comunidade acadêmica da Universidade Federal do Pampa - Campus 
Itaqui, que aderiram livremente à pesquisa, mediante assinatura do Termo de Consentimento Livre e Esclarecido. No teste de aceitabilidade, os muffins foram oferecidos a 40 provadores, um por vez e de maneira aleatória, para que avaliassem os atributos de cor, odor, sabor e textura, a partir de escala hedônica estruturada de nove pontos, sendo 1 para "desgostei muitíssimo" e 9 para "gostei muitíssimo". Com as médias do teste de aceitabilidade, calculou-se o índice de aceitabilidade (IA) através da seguinte fórmula: $\mid A=(A \times 100) / B$, em que $A=$ média das notas atribuídas pelos provadores e $B=$ nota máxima atribuída ao produto. O teste de intenção de compra foi realizado a partir de escala hedônica estruturada de cinco pontos, sendo 1 para "certamente não compraria" e 5 para "certamente compraria". No teste de ordenação de preferência, outros 40 provadores receberam as cinco formulações de muffins ao mesmo tempo, para que ordenassem da esquerda para a direita, da amostra "menos preferida" até a "mais preferida".

\subsection{Análise dos resultados}

O experimento foi conduzido em delineamento inteiramente casualizado. Através do programa Statistica, versão 8.0, os dados foram submetidos à análise de variância e as médias comparadas pelo teste de Tukey, em nível de $5 \%$ de significância. Os dados do teste de ordenação de preferência foram submetidos ao Teste de Friedman e as diferenças, significativas ou não, determinadas através da Tabela de Newell e MacFarlane (IAL, 2008).

\section{Resultados e discussão}

\subsection{Análises físicas}

As propriedades físicas relacionadas a peso, rendimento e volume dos muffins estão apresentadas na Tabela 2.

Os rendimentos dos muffins com farinha de feijão foram significativamente $(P<0,05)$ superiores ao MP. Da mesma forma, a perda de peso do MP foi significativamente $(P<0,05)$ superior aos muffins com farinha de feijão. Tais resultados sugerem que as farinhas de feijão conferiram maior capacidade de retenção de água, a qual foi mantida na massa durante o assamento, provavelmente devido à interação com seus componentes, tais como proteínas, amido e, especialmente, fibras. No entanto, as diferenças de rendimento e perda de peso não proporcionaram muffins visualmente maiores, pois os produtos não diferiram significativamente $(P>0,05)$ no volume aparente e específico.

Alguns trabalhos descrevem resultados semelhantes aos apresentados. Bibalan et al. (2013) constataram maior absorção de água em muffins com inclusão de farelo de arroz e atribuíram o fato ao incremento dos teores de fibra alimentar nos produtos. Froes (2012) descreveu que quanto maior a proporção de farinha de bandinha de feijão carioca em mistura para bolo, maior a absorção de água. Leal e Almeida (2016) constataram valores de volume específico entre 1,66 e 2,10 $\mathrm{mL} \mathrm{g}^{-1}$ em muffins elaborados com diferentes sistemas de levedação. Já Segura et al. (2014), na elaboração de muffins com farinha de arroz e diferentes níveis de goma xantana, também não observaram diferenças no volume dos produtos.

A Tabela 1 apresenta os custos das formulações, tanto para a quantidade de $367 \mathrm{~g}$ de massa como para as unidades dos muffins. A substituição da farinha de trigo pelas farinhas de feijão acarretou maiores custos das massas cruas, proporcionalmente ao valor comercial de cada classe de feijão. Entretanto, após o processo de assamento e considerados rendimentos e pesos finais (Tabela 2), os custos das unidades de muffins foram semelhantes, com valor médio de $\mathrm{R} \$ 0,08$, sem considerar custos com energia elétrica, mão de obra, embalagem e impostos. Tal constatação demonstra que a substituição da farinha de trigo por farinhas de feijão não encareceu o muffin.

\subsection{Análises químicas}

$\mathrm{Na}$ Tabela 3, são apresentados os resultados da composição química, do valor calórico e do teor de compostos fenólicos totais das formulações de muffins.

Em relação ao teor de umidade, observa-se uma relação com o rendimento e a perda de peso descritos

Tabela 2. Propriedades físicas das formulações de muffins.

\begin{tabular}{|c|c|c|c|c|c|}
\hline Muffin & $\begin{array}{c}\text { Peso massa crua } \\
\text { (g) }\end{array}$ & $\begin{array}{c}\text { Peso massa cozida } \\
\text { (g) }\end{array}$ & $\begin{array}{c}\text { Rendimento em } \\
\text { peso }\end{array}$ & $\begin{array}{c}\text { Perda de peso } \\
(\%)\end{array}$ & $\begin{array}{l}\text { Volume específico } \\
\qquad\left(m L ~^{-1}\right)\end{array}$ \\
\hline MP & $21,39 \pm 1,67 a$ & $18,15 \pm 1,44 a b$ & $0,85 \pm 0,01 c$ & $15,14 \pm 1,17 \mathrm{a}$ & $1,89 \pm 0,40 a$ \\
\hline MFB & $19,08 \pm 0,96 b$ & $16,81 \pm 0,86 b$ & $0,88 \pm 0,01 b$ & $11,91 \pm 0,81 b$ & $1,87 \pm 0,29 a$ \\
\hline MFV & $19,99 \pm 1,68 a b$ & $17,58 \pm 1,52 a b$ & $0,88 \pm 0,01 b$ & $12,07 \pm 0,64 b$ & $2,01 \pm 0,41 \mathrm{a}$ \\
\hline MFC & $21,02 \pm 0,47 a$ & $18,79 \pm 0,43 a$ & $0,89 \pm 0,01 a$ & $10,59 \pm 0,33 c$ & $1,91 \pm 0,32 a$ \\
\hline MFP & $19,25 \pm 0,74 b$ & $17,15 \pm 0,82 b$ & $0,89 \pm 0,01 a b$ & $10,91 \pm 1,51 b c$ & $2,16 \pm 0,33 a$ \\
\hline
\end{tabular}

Valores expressos como média \pm desvio padrão seguidos por letras distintas que indicam diferença estatística significativa nas linhas, em nível de $5 \%$ pelo teste de Tukey. Legenda: MP (muffin padrão), MFB (muffin feijão branco), MFV (muffin feijão vermelho), MFC (muffin feijão carioca) e MFP (muffin feijão preto). 
na Tabela 2, pois os produtos de maiores rendimentos e menores perdas de peso apresentaram maior umidade. O MP apresentou menor teor de umidade em relação aos muffins com farinha de feijão, que diferiram entre si com menor teor de umidade no MFV, umidade intermediária no MFB e maior teor de umidade nos MFC e MFP. As diferenças observadas provavelmente estão relacionadas à capacidade de retenção de água dos componentes da farinha de feijão, especialmente da fibra alimentar, componente que mais aumentou com a inclusão das farinhas de feijão (Tabela 3).

A matéria mineral, correspondente à fração cinzas, também foi significativamente superior nos muffins com farinha de feijão, em relação ao MP (Tabela 4). O incremento de minerais pode ser explicado pela composição das farinhas utilizadas, pois o feijão tem, pelo menos, 3,5\% de cinzas na composição, enquanto que a farinha de trigo tem apenas 0,8\% (UNICAMP, 2011). Dos minerais presentes no feijão, destacam-se quantitativamente potássio (mais de 1\%), fósforo (cerca de 0,4\%), ferro (cerca de 0,007\%), além de magnésio, cálcio, zinco, manganês e cobre, apenas com quantidades traço de sódio (LAJOLO et al., 1996; UNICAMP, 2011).
O conteúdo de lipídeos não diferiu $(P>0,05)$ entre os muffins (Tabela 3). Tal resultado pode ser atribuído à composição das farinhas utilizadas, pois tanto a farinha de trigo como as farinhas de feijão têm baixo conteúdo lipídico, 1,4\% e cerca de 1,3\%, respectivamente, conforme tabelas de composição de alimentos (DIS, 2015; UNICAMP, 2011). No entanto, o teor de lipídeos dos muffins elaborados não foi baixo, com médias variando de 13,09\% a 13,91\%, decorrente dos demais ingredientes da composição, principalmente das gemas dos ovos e do óleo de soja.

O conteúdo proteico dos muffins com substituição da farinha de trigo também aumentou significativamente $(P<0,05)$ em MFB, MFV e MFP, em relação ao MP; apenas o MFC não diferiu significativamente do MP $(P>0,05)$ (Tabela 3). Esse resultado pode ser atribuído ao superior teor proteico dos feijões em relação à farinha de trigo, mais de $20 \%$ e de 9,8\%, respectivamente (UNICAMP, 2011). Entre os muffins, o menor conteúdo proteico do MFC é decorrente do maior teor de proteínas no feijão carioca (20\%) em relação aos demais utilizados (UNICAMP, 2011); 23,36\% para o feijão branco e 21,3\% (DIS, 2015) para o preto (UNICAMP, 2011). Esta possibilidade é reforçada considerando-se o trabalho de Marquezi (2013), que

Tabela 3. Composição química e valor calórico das formulações de muffins.

\begin{tabular}{|c|c|c|c|c|c|}
\hline Componente (\%) & MP & MFB & MFV & MFC & MFP \\
\hline Umidade & $21,81 \pm 0,01 d$ & $23,98 \pm 0,07 b$ & $23,32 \pm 0,04 c$ & $25,06 \pm 0,15 a$ & $25,15 \pm 0,10 a$ \\
\hline Matéria mineral & $1,16 \pm 0,03 b$ & $1,28 \pm 0,03 a$ & $1,29 \pm 0,03 a$ & $1,28 \pm 0,04 a$ & $1,27 \pm 0,03 a$ \\
\hline Proteínas & $10,17 \pm 0,04 b$ & $11,52 \pm 0,15 a$ & $11,96 \pm 0,24 a$ & $10,38 \pm 0,12 b$ & $11,39 \pm 0,51 a$ \\
\hline Fibra alimentar & $3,71 \pm 0,17 \mathrm{e}$ & $7,63 \pm 0,10 \mathrm{c}$ & $9,12 \pm 0,62 b$ & $6,25 \pm 0,06 \mathrm{~d}$ & $9,92 \pm 0,10 a$ \\
\hline Insolúvel & $3,68 \pm 0,17 c$ & $5,84 \pm 0,41 a$ & $4,09 \pm 0,17 \mathrm{c}$ & $5,17 \pm 0,19 b$ & $5,25 \pm 0,06 a b$ \\
\hline Solúvel & $0,03 \pm 0,01 \mathrm{c}$ & $1,80 \pm 0,31 b$ & $5,03 \pm 0,47 a$ & $1,08 \pm 0,13 b$ & $4,67 \pm 0,16 a$ \\
\hline Carboidratos & $49,25 \pm 0,31 a$ & $42,09 \pm 0,34 b c$ & $40,72 \pm 0,97 \mathrm{~cd}$ & $43,70 \pm 0,54 b$ & $39,18 \pm 0,96 d$ \\
\hline $\begin{array}{l}\text { Valor calórico } \\
\left(\text { kcal } 100 \mathrm{~g}^{-1}\right)\end{array}$ & $362,85 \pm 2,63 a$ & $335,89 \pm 1,81 b$ & $332,98 \pm 2,59 b$ & $337,12 \pm 2,57 b$ & $320,09 \pm 1,59 c$ \\
\hline $\begin{array}{l}\text { Compostos fenólicos } \\
\left(m g \text { AGE* } 100 \mathrm{~g}^{-1}\right)\end{array}$ & $19,16 \pm 2,16 c$ & $30,34 \pm 2,04 b$ & $33,77 \pm 1,04 a b$ & $32,71 \pm 1,49 a b$ & $37,06 \pm 2,28 a$ \\
\hline
\end{tabular}

Valores expressos como média \pm desvio padrão seguidos por letras que indicam diferença estatística significativa nas linhas, em nível de 5\% pelo teste de Tukey. *AGE (Equivalente ácido gálico); Legenda: MP (muffin padrão), MFB (muffin feijão branco), MFV (muffin feijão vermelho), MFC (muffin feijão carioca) e MFP (muffin feijão preto).

Tabela 4. Análise sensorial de aceitação e intenção de compra dos muffins.

\begin{tabular}{cccccc} 
Teste de aceitabilidade & MP & MFB & MFV & MFC \\
Cor & $8,32 \pm 0,76 \mathrm{a}$ & $8,34 \pm 0,76 \mathrm{a}$ & $7,76 \pm 1,37 \mathrm{a}$ & $7,73 \pm 1,22 \mathrm{a}$ & $7,73 \pm 1,28 \mathrm{a}$ \\
Odor & $8,10 \pm 0,89 \mathrm{ab}$ & $8,22 \pm 0,82 \mathrm{a}$ & $7,44 \pm 1,40 \mathrm{~b}$ & $7,80 \pm 1,21 \mathrm{ab}$ & $7,76 \pm 1,30 \mathrm{ab}$ \\
Sabor & $8,32 \pm 0,82 \mathrm{a}$ & $8,02 \pm 1,29 \mathrm{ab}$ & $7,29 \pm 1,58 \mathrm{~b}$ & $7,51 \pm 1,53 \mathrm{ab}$ & $8,05 \pm 1,02 \mathrm{ab}$ \\
Textura & $8,39 \pm 0,77 \mathrm{a}$ & $7,98 \pm 1,23 \mathrm{ab}$ & $7,66 \pm 1,44 \mathrm{ab}$ & $7,49 \pm 1,67 \mathrm{~b}$ & $7,85 \pm 1,35 \mathrm{ab}$ \\
Índice de aceitabilidade & $92,00 \%$ & $90,44 \%$ & $83,78 \%$ & $84,78 \%$ & $87,22 \%$ \\
Teste de Intenção de compra & $4,66 \pm 0,48 \mathrm{a}$ & $4,39 \pm 0,92 \mathrm{ab}$ & $3,93 \pm 0,98 \mathrm{~b}$ & $4,07 \pm 0,88 \mathrm{~b}$ & $4,41 \pm 0,77 \mathrm{ab}$ \\
\hline
\end{tabular}

Valores expressos como média \pm desvio padrão seguidos por letras que indicam diferença estatística significativa nas linhas, em nível de $5 \%$ pelo teste de Tukey. Legenda: MP (muffin padrão), MFB (muffin feijão branco), MFV (muffin feijão vermelho), MFC (muffin feijão carioca) e MFP (muffin feijão preto). 
descreve a composição de diferentes cultivares de feijão, com menores teores proteicos no feijão carioca em relação aos feijões vermelho e preto.

Quanto ao teor de fibra alimentar, o MP apresentou menor valor em comparação aos muffins com feijão, dos quais o maior valor foi constatado no MFP, seguido, de modo decrescente, por MFV, MFB e MFC (Tabela 3). $O$ incremento de fibra alimentar nos muffins com substituição da farinha de trigo pelas farinhas de feijões era esperado, considerando, por exemplo, teores relatados para feijão carioca e preto de respectivos $18,4 \%$ e $21,8 \%$, enquanto que, para a farinha de trigo, é relatado um teor de apenas 2,3\% do nutriente (UNICAMP, 2011).

Em relação ao tipo de fibra, MFV e MFP se destacaram pelos maiores teores de fibra alimentar solúvel, enquanto que MP, MFB e MFC apresentaram maior proporção de fibra insolúvel (Tabela 3 ).

A presença das fibras alimentares do feijão na dieta proporciona benefícios ao trato gastrointestinal desde sua ingestão até sua excreção, diminuindo a incidência de problemas como constipação, diarreia e câncer colorretal. As fibras insolúveis proporcionam o aumento do volume fecal, diminuindo o tempo de trânsito intestinal, aumentando a sensação de saciedade do organismo, reduzindo o tempo de absorção da glicose e promovendo a eliminação de resíduos que se alojam na parede intestinal. As fibras solúveis desenvolvem uma massa viscosa durante a passagem pelo intestino, que aumenta o conteúdo do estômago, atrasando o esvaziamento gástrico, proporcionando maior volume e lubrificação fecal, e reduzindo os níveis de colesterol plasmático e resposta glicêmica (CÁRDENAS et al., 2010).

Os incrementos nos teores de umidade, cinzas, proteínas e fibras nas formulações de muffins que tiveram a farinha de trigo parcialmente substituída pelas farinhas de feijões resultaram em menores valores no conteúdo de carboidratos digeríveis. O MP apresentou valor significativamente superior de carboidratos digeríveis e valor calórico. Entre os muffins com farinha de feijão, o MFP apresentou menor conteúdo de carboidratos digeríveis e, consequentemente, menor valor calórico (Tabela 3).

Outros trabalhos com substituição da farinha de trigo em muffins ou inclusão de farinha de feijão em diversos produtos de panificação também reportam incrementos nos teores de umidade, cinzas, proteínas e fibra alimentar, em detrimento dos carboidratos digeríveis e valor calórico (FROES, 2012; FROTA et al., 2010; KUNTZ, 2013).

Quanto aos teores de compostos fenólicos totais, os mesmos foram maiores no MFP, seguido do MFV e MFC, os quais não diferiram significativamente $(P>0,05)$ do MFP e MFB (Tabela 3). A variação nos teores destes compostos pode ser explicada pelos níveis dos mesmos no tegumento dos feijões utilizados para elaboração das farinhas. Feijões com tegumento mais escuro apresentam maiores teores de taninos e, por consequência, maior teor de compostos fenólicos totais do que aqueles com tegumentos claros (ESTEVES et al., 2002; MESQUITA et al., 2007). Em feijões marrons, pretos, vermelhos e brancos, o teor médio de taninos é de 7,8; 6,6; 12,6 e 2,3 $\mathrm{mg} \mathrm{g}^{-1}$ de equivalentes de catequina, respectivamente (LAJOLO et al., 1996). Dessa forma, pode-se inferir que o mesmo comportamento seja verificado nos produtos elaborados com a farinha desses feijões, presumindo que aqueles alimentos que contenham farinha de feijão de tegumento mais escuro apresentem maior teor de compostos fenólicos.

Como já demonstrado, os muffins são produtos com consideráveis teores de proteínas. Tal fato pode ter sido um interferente positivo na determinação dos compostos fenólicos, visto que não foi realizada uma precipitação das proteínas presentes nesses alimentos antes da quantificação dos mesmos. O método espectrofotométrico de Folin Ciocalteau não é um método específico, pois a reação, além de envolver os grupamentos fenólicos presentes no meio, também pode envolver substâncias redutoras presentes nos alimentos, como aminoácidos e proteínas, interferindo positivamente nos resultados (ANGELO; JORGE, 2007). Entretanto, caso o mencionado tenha ocorrido, a interferência ocorreu em todas as amostras analisadas, não prejudicando o comparativo entre as mesmas.

Os compostos fenólicos de maior destaque no feijão são as antocianinas e os taninos, cuja incidência pode influenciar na cor, no aroma e na qualidade nutricional desta leguminosa (BARRUETO-GONZALEZ, 2008). Além disso, é importante destacar que, ao consumo regular de compostos fenólicos, estão atribuídos diversos benefícios para a saúde, como efeitos anticarcinogênicos, por meio de mecanismo antioxidante de neutralização de radicais livres (CÁRDENAS et al., 2010).

\subsection{Análises sensoriais}

$\mathrm{Na}$ Tabela 4, estão expostos os resultados dos atributos avaliados pelos provadores nos testes de aceitabilidade e intenção de compra.

No teste de aceitabilidade, apenas no atributo cor, os muffins não diferiram $(P>0,05)$. Quanto ao odor, o MFB foi mais e o MFV menos aceito, enquanto que os demais não diferiram. No sabor, o MP foi mais e o MFV, novamente, menos aceito, sem diferença para os demais muffins. Na textura, o MP foi mais e o MFC menos aceito, também sem diferença para os demais produtos. De modo geral, os resultados do teste de aceitabilidade demonstram o MP mais aceito pelos provadores, mas com aceitação equivalente $(P>0,05)$ a MFB e MFP em todos os atributos avaliados. Ressalta-se que todas as médias das notas atribuídas pelos provadores no teste de aceitabilidade foram superiores a 7 na escala hedônica, demonstrando 
que os provadores, ao menos, "gostaram regularmente" dos muffins.

Para um produto ser considerado "aceitável", deve apresentar, no mínimo, 70\% de aceitabilidade (DUTKOSKY, 2007). De acordo com os índices descritos na Tabela 4 , todos os muffins superaram o valor considerado "aceitável", com menor e maior índice de aceitabilidade observados no MFV e MP, respectivamente.

Diversos trabalhos descrevem boa aceitação para o consumo de muffins. Souza et al. (2014) obtiveram altas notas para os atributos cor, aroma, textura, sabor e impressão global de muffins adicionados de castanha portuguesa. Mosca et al. (2014) observaram notas de aceitação global superiores a 4 (gostei), em uma escala hedônica facial de cinco pontos, para bolo de chocolate adicionado de feijão carioca cozido, tendo crianças de uma creche/escola como provadores. Frota et al. (2010) constataram boa aceitação global em formulações de biscoitos e rocamboles com feijão-caupi, com notas superiores a 6 , correspondente a "gostei ligeiramente", em escala hedônica de nove pontos. Da mesma forma, Bender (2015) teve índices de aceitabilidade superiores a $70 \%$ em muffins adicionados de casca de uva concentrada em fibra.

O teste de intenção de compra reproduziu a aceitabilidade dos provadores, com maior intenção de compra pelo MP, sem diferir do MFB e MFP, enquanto que o MFV e MFC tiveram notas menores. Ressalta-se que as médias das notas para o MFV foram superiores ou próximas de 4, que corresponde à "provavelmente compraria" na escala hedônica de intenção de compra (Tabela 4).

A Tabela 5 demonstra o resultado do teste de ordenação de preferência dos muffins, através do somatório de pontos. O MP foi mais preferido, enquanto que o MFV foi menos preferido pelos provadores; os demais, MFB, MFC e MFP, não diferiram significativamente $(P>0,05)$ do MP e MFV.

Mosca et al. (2014) constataram preferência equivalente em bolo de chocolate com e sem adição de feijão carioca cozido, através do teste pareado de preferência, tendo crianças de uma creche/escola como provadores.

Tabela 5. Somatório de pontos dos muffins no teste de ordenação de preferência.

\begin{tabular}{cccccc} 
Muffin & MP & MFB & MFV & MFC & MFP \\
$\begin{array}{l}\text { Somatório } \\
\text { de pontos }\end{array}$ & $153 \mathrm{a}$ & $117 \mathrm{ab}$ & $97 \mathrm{~b}$ & $132 \mathrm{ab}$ & $116 \mathrm{ab}$ \\
\hline
\end{tabular}

Valores seguidos por letras distintas indicam diferença estatística significativa em nível de 5\% pelo teste de Friedman. Legenda: MP (muffin padrão), MFB (muffin feijão branco), MFV (muffin feijão vermelho), MFC (muffin feijão carioca) e MFP (muffin feijão preto).

\section{Conclusão}

A adição de farinhas de diferentes classes de feijão, em substituição à farinha de trigo, alterou as propriedades físicas relacionadas ao peso dos produtos. Nutricionalmente, admite-se o incremento do valor nutricional devido ao aumento dos teores de matéria mineral, proteína, fibra alimentar e compostos fenólicos totais, em detrimento dos carboidratos digeríveis e do valor calórico. Sensorialmente, os muffins com substituição de $30 \%$ da farinha de trigo por farinha de feijão branco e preto tiveram aceitação, intenção de compra e preferência equivalentes ao muffin padrão. Embora os muffins com farinha de feijão vermelho e carioca tenham obtido menor aceitação nos atributos odor, sabor textura, além de menor intenção de compra, e, no caso do muffin de feijão vermelho, menor preferência dos provadores, todos os muffins apresentaram altos índices de aceitabilidade. De modo geral, o muffin com farinha de feijão preto destacou-se pelo maior valor nutricional e pela equivalência ao muffin padrão, em relação às propriedades físicas e atributos sensoriais.

Esses resultados são de grande relevância para a indústria de alimentos, pois evidenciam que a substituição parcial de ingredientes energéticos, como a farinha de trigo, por farinha de feijão possibilita obter muffins com propriedades físicas adequadas, melhor qualidade nutricional e altos índices de aceitabilidade, sem encarecer o produto final.

\section{Referências}

AMERICAN ASSOCIATION OF CEREAL CHEMISTS INTERNATIONAL - AACCI. 11th ed. Approved methods of analysis: method 10-05.01. guidelines for measurement of volume by rapeseed displacement. Minnesota: AACC International, 2001.

ANGELO, P. M.; JORGE, N. Compostos fenólicos em alimentos: uma breve revisão. Revista do Instituto Adolfo Lutz, v. 66, n. 1, p. 1-9, 2007.

ASSOCIATION OF OFFICIAL ANALYTICAL CHEMISTS - AOAC. Official methods of analysis. 17th ed. Gaithersburg: AOAC, 2000.

BARCELO, D. M. S.; ANTÔNIO, L. C.; RODRIGUES, J. P. M.; OLIVEIRA, L. F. Processamento e análise sensorial de bolo de chocolate com farelo de mandioca desidratado. Revista Faculdade Montes Belos, v. 7, n. 1, p. 14-129, 2014.

BARCHET, G. V.; MATTOS, K. M.; LIMA, L. D.; SOMAVILLA, M.; ROCHA, T. S.; BORTOLUZZI, V. P. Análise sensorial de bolo enriquecido com feijão: a aceitabilidade por pré-escolares. Revista Digital, v. 15, n. 144, 2010. Disponível em: <http://www. efdeportes.com/efd144/analise-sensorial-de-bolo-enriquecidocom-feijao.htm>. Acesso em: 22 jun. 2015.

BARRUETO-GONZALEZ, N. B. Biodisponibilidade de minerais das fontes leguminosas. Revista Simbio-Logias, v. 1, n. 1, p. 174-183, 2008. 
Muffins adicionados de farinha de feijão de diferentes classes

Barros, L. F. T et al.

BenAssi, V. T.; WATANABE, E.; LOBO, A. R. Produtos de panificação com conteúdo calórico reduzido. Boletim do Centro de Pesquisa de Processamento de Alimentos, v. 19, n. 2, p. 225-242, 2001

BENDER, A. B. B. Fibra alimentar a partir de casca de uva: desenvolvimento e incorporação em bolos tipo muffins. 2015. 132 f. Dissertação (Mestrado em Ciência e Tecnologia dos Alimentos) - Universidade Federal de Santa Maria, Santa Maria, 2015.

BENNEMANN, G. D.; NEZELLO, M. C.; EING, K. K. C.; NOVELLO, D.; SCHWARZ, K.; BOTELHO, R. V. Desenvolvimento e aceitabilidade de muffins adicionados de farinha de casca de uva das cultivares Ancelotta e Bordô. Revista da Universidade Vale do Rio Verde, v. 14, n. 2, p. 864-874, 2016.

BIBALAN, S. G.; SALEHLI, E. A.; SANI, A. M. An investigation on the effect of rice bran addition on the paste rheological characteristics and chemical composition of muffin cake. Journal of Innovation in Food Science and Technology, Sabzevar, v. 5, n. 2, p. 1-7, 2013.

BLIGH, E. G.; DYER, W. J. A rapid method of total lipid extraction and purification. Canadian Journal of Biochemistry and Physiology, v. 37, n. 8, p. 911-917, 1959. http://dx.doi.org/10.1139/ o59-099. PMid:13671378.

BRASIL. Ministério da Saúde. Agência Nacional de Vigilância Sanitária. Resolução RDC n 360, de 23 de dezembro de 2003. Aprova Regulamento Técnico sobre Rotulagem Nutricional de Alimentos Embalados, tornando obrigatória a rotulagem nutricional. Diário Oficial [da] República Federativa do Brasil, Brasília, DF, 26 dez. 2003.

CÁRDENAS, L. L. A. R.; ROSA, C. O. B.; COSTA, N. M. B. Propriedades funcionais do feijão. In: COSTA, N. M. B.; ROSA, C. O. B. Alimentos funcionais: componentes bioativos e efeitos fisiológicos. Rio de Janeiro: Editora Rubio, 2010. cap. 10, p. 157-176.

CASAGRANDI, D. A.; CANNIATTI-BRAZACA, S. G.; SALGADO, J. M.; PIZZINATO, A.; NOVAES, N. J. Análise tecnológica, nutricional e sensorial de macarrão elaborado com farinha de trigo adicionada de farinha de feijão-guandu. Revista de Nutrição, v. 12, n. 2, p. 137-143, 1999. http://dx.doi.org/10.1590/ S1415-52731999000200003.

COMPANHIA NACIONAL DE ABASTECIMENTO - CONAB. Conjuntura agropecuária do feijão. João Pessoa: CONAB, 2015. Disponível em: <http://www.conab.gov.br/OlalaCMS/uploads/ arquivos/15_07_09_16_20_14_conjuntura_agropecuaria_do_ feijao_-_junho_2015.pdf>. Acesso em: 17 out. 2017.

CURREA, A. M.; SIERRA, A. F. Naffins: muffins \& cupcakes. 2010. 54 f. Trabalho de Conclusão de Curso (Carrera de Diseño Industrial)-Pontificia Universidad Javeriana, Bogotá, 2010.

DEPARTAMENTO DE INFORMÁTICA EM SAÚDE - DIS. Escola Paulista de Medicina - EPM-UNIFESP. Tabela de composição química dos alimentos - TABNUT. 2015. Disponível em: <http:// www2.unifesp.br/dis/servicos/nutri/public/>. Acesso em: 14 jun. 2015.

DUTKOSKY, S. D. Análise sensorial de alimentos. 2. ed. Curitiba: Champagnat, 2007. 239 p.

ESTEVES, M. A.; ABREU, C. M. P.; SANTOS, C. D.; CORRÊA, A. D. Comparação química e enzimática de seis linhagens de Feijão (Phaseolus vulgaris L.). Ciência e Agrotecnologia, v. 26, n. 5, p. 999-1005, 2002.

FOOD AND AGRICULTURE ORGANIZATION OF THE UNITED NATIONS - FAO. FAOSTAT Statistics Division. Roma: FAO, 2014. Disponível em: <http://www.fao.org/faostat/en/\#data/QC>. Acesso em: 17 out. 2017

FROES, L. O. Emprego da farinha de bandinha de feijão carioca extrusada na formulação de misturas para bolo sem glúten contendo farinha de quirera de arroz. 2012. 122 f. Dissertação (Mestrado em Ciência e Tecnologia de Alimentos) - Escola de Agronomia e Engenharia de Alimentos, Universidade Federal de Goiás, Goiânia, 2012.

FROTA, K. M. G.; MORGANO, M. A.; SILVA, M. G.; ARAÚJO, M. A. M.; MOREIRA-ARAÚJO, R. S. R. Utilização da farinha de feijão-caupi (Vigna unguiculata L. Walp) na elaboração de produtos de panificação. Ciência e Tecnologia de Alimentos, v. 30, n. 1, p. 44-50, 2010.

INSTITUTO ADOLFO LUTZ - IAL. Métodos físico-químicos para análise de alimentos. 4. ed. São Paulo: IAL, 2008.

KUNTZ, M. G. F. Efeitos da inulina nas propriedades físicas, químicas, de textura e aceitabilidade no desenvolvimento de muffin destinado a consumidores em idade escolar. 2013. 150 f. Tese (Doutorado em Ciência de Alimentos) - Programa de Pós-Graduação em Ciência e Tecnologia de Alimentos, Universidade Federal de Santa Catarina, Florianópolis, 2013.

LAJOLO, F. M.; GENOVESE, M. I.; MENEZES, E. W. Qualidade nutricional. In: ARAÚJO, R. S.; RAVA, C. A.; STONE, L. F.; ZIMMERMANN, M. J. O. Cultura do feijoeiro comum no Brasil. Piracicaba: POTAFOS, 1996.

LEAL, A. R. S.; ALMEIDA, E. L. Influência do sistema de levedação em muffins assados em forno de micro-ondas. In: CONGRESSO BRASILEIRO DE CIÊNCIA E TECNOLOGIA DE ALIMENTOS, 25., 2016, Gramado. Anais... Campinas: SBCTA, 2016. Disponível em: <http://www.ufrgs.br/sbctars-eventos/xxvcbcta/anais/files/1127. pdf>. Acesso em: 17 outubro 2017.

MARQUEZI, M. Características físico-químicas e avaliação das propriedades tecnológicas do feijão comum (Phaseolus vulgaris L.). 2013. 115 f. Dissertação (Mestrado em Ciência de Alimentos) - Programa de Pós-Graduação em Alimentos, Universidade Federal de Santa Catarina, Florianópolis, 2013.

MARTÍNEZ-CERVERA, S.; SANZ, T.; SALVADOR, A.; FISZMAN, S. $M$. Rehological, textural and sensorial properties os low-sucrose muffins reformulated with sucralose/polydextrose. Lebensmittel- 
Wissenschaft + Technologie, v. 45, n. 2, p. 213-220, 2012. http://dx.doi.org/10.1016/j.Iwt.2011.08.001.

MESQUiTA, F. R.; CORRÊA, A. D.; ABREU, C. M. P.; LIMA, R. A. Z.; ABREU, A. F. B. Linhagens de feijão (Phaseolus vulgaris L.): composição química e digestibilidade proteica. Ciência e Agrotecnologia, v. 31, n. 4, p. 1114-1121, 2007.

MOSCA, P. C.; OLIVEIRA, F. M.; ZANARDO, F. C.; ROSSATO, F. M.; ALMEIDA, J. M.; PEREIRA, E. M. S.; TAVANO, O. L. Composição e aceitabilidade entre crianças de uma creche/ escola de Uberaba-MG de bolo de chocolate adicionado de grãos de feijão cozidos. Revista Brasileira de Produtos Agroindustriais, v. 16, n. 4, p. 403-410, 2014.

MOSCATTO, A. J.; PRUDÊNCIO-FERREIRA, H. S.; HAULY, M. C. $O$. Farinha de yacon e inulina como ingredientes na formulação de bolo de chocolate. Ciência e Tecnologia de Alimentos, v. 24, n. 4, p. 634-640, 2004.

POQUETTE, N. M.; GU, X.; LEE, S.-O. Grain sorghum muffin reduces glucose and insulin responses in men. Food \& Function, v. 5 , n. 5, p. 894-899, 2014. http://dx.doi.org/10.1039/C3FO60432B. PMid:24608948.

SEGURA, M. E. M.; SANZ, T.; FISZMAN, S.; ROSELL, C. M. Development and quality of rice flour-based gluten-free muffins. Caracas: Instituto de Agroquímica y Tecnología de Alimentos, 2014. Disponível em: <http://digital.csic.es/ handle/10261/79677?mode=full\& su-bmit_simple=Mostrar+el
+registro+Dublin+Core+completo+del+\%C3\%ADtem+\%C2\%B B>. Acesso em: 18 jun. 2015.

SILVA, A. G.; ROCHA, L. C.; BRAZACCA, S. G. C. Caracterização físico-química. digestibilidade proteica e atividade antioxidante de feijão. Alimentos e Nutrição, v. 20, n. 4, p. 591-598, 2009.

SINGLETON, V. L.; ROSSI, J. A. Jr. Colorimetry of total phenolics with phosphomolybdic-phosphotungstic acid reagents. American Journal of Enology and Viticulture, Modesto, v. 16, p. 144-158, 1965.

SOUZA, A. G.; SANTOS, L. S.; SILVA, A. R. Z.; PASSONI, C. R. M. S. Propriedades nutricionais da castanha portuguesa (Castanea sativa Mill) e elaboração de produtos. Cadernos da Escola de Saúde, v. 2, n. 12, p. 109-124, 2014.

UNIVERSIDADE ESTADUAL DE CAMPINAS - UNICAMP. TACO: Tabela brasileira de composição de alimentos. 4. ed. Campinas: NEPA; UNICAMP, 2011. Disponível em: <http://www.cfn.org.br/ wp-content/uploads/2017/03/taco_4_edicao_ampliada_e_revisada. pdf>. Acesso em: 18 jun. 2015.

VELIOGLU, Y. S.; MAZZA, G.; GAO, L.; OOMAH, B. D. Antioxidant activity and total phenolics in selected fruits, vegetables, and grain products. Journal of Agricultural Food Chemistry, Washington, v. 46, n. 10, p. 4113-4117, 1998. http://dx.doi. org/10.1021/jf9801973.

VIEIRA, C.; PAULA JÚNIOR, T. J.; BORÉM, A. Feijão. 2. ed. Viçosa: Editora UFV, 2011. 600 p. 